\title{
Alpha Particle Confinement in Tokamaks
}

\author{
R.B. White and H.E.M!ynick \\ Plasma Physics Laboratory. Princeton Lniversity \\ P.O. Bux 451 \\ Princeton, Sew Jersey 08543-0451, L.S.A.
}

\begin{abstract}
An assessment of diffusive tokamak transport mechanisms of concern for alpha particles indicates that the "stochastic regime" is the only one which appears to pose a real danger for adequate alpha confinement. This fact, in conjunction with the threshold character of that mechanism, allows one to decide whether an alpha born at a given location will be lost or confined, according to a very simple criterion. Implementing this criterion numerically results in a new code for the assessment of alpha confinement, which is orders of magnitude faster than earlier codes used for this purpose.
\end{abstract}


Achieving ignition in tokamaks depends critucally on these machines confining the alpha particles produced bv the D- $T$ fusion reaction long enough that their energy gres into keeping the plasma at fusion remperatures. Iokamaks are now entering a dontain of uperation where the loss rates of alpha (ur alpha-like) fusion products can be measured. and a good theuretical tunderstanding of these loss rates is essential in designing future machines. In this work, we describe progress we have made in achieving the needed theoretical understanding.

The progress is embodied in a new code (HIPLOS) for calculating tokamak aipha loss, which is much faster than both the guding-center $(\mathrm{gc})^{1-3}$ and the "mapping-method" ( $\mathrm{mm})^{3,5}$ codes which up to now have provided the best numerical tools for assessing the transport, and whose results are in reasonable agreement with the results of the gc codes in the benchnark cases run thus far. For the guiding-center code, a typical ensemble consists of 500 particles. and takes several hours on the Cray, while for RIPLOS, an ensemble of 40,1111$)$ particles takes only about 1 minute, implying a boost in the speed per particle between the two codes of order $10^{4-5}$. The code's simplicitv (and thus speed) is made possible by two conceptual steps. First, an assessment of the existing body of analytic theory of diffusive mecharisms of possible concern for alpha transport indicates that, of all the potential candidales. the onlv mechanism which really appears to pose a problem for alpha confinement is the "stochastic regime" of ripple transport, generalized ${ }^{\top}$ to apply to the ripple generated by low-n MHD activity, as weii as to that from toroidal-field (TF) coils, for which the original theory was developed. This simplification allows one to focus exclusively on the particular properties of stochastic cipple transport. Second, ihis stochastic mechanism has a threshold character spatiallv. and this fact permits us to distill a very simple criterion for deciding whether an alpha borm at a given location will be adequately confined or not. The implementation of this criterion results in the RIPLOS code.

We first review the various diffusive mechanisms which have been considered analytically. Table I summarizes the assessment. Each mechanism is described by a radial diffusion coefficient $D$, which, using th: approximate formuls $\tau_{a}=a^{2} /(+D)$ ( $a$ is the tokamak minor radius), yields the alpha confinement time $\tau_{a}$. "Acceptable confinement" of the alphas then holds when $\tau_{a} \geq \tau_{x}$, with $\tau_{\text {,l }}$ the slowing-down time. For definiteness. we shall evaluate the various $D$ 's at $r=a / 2$ for a particular set of INTOR parameters: $R=5.3 \mathrm{~m}, a=1.0 \mathrm{~m}, B=5.5 \mathrm{~T}, n_{e}(a / 2)=$ 
$1.5 \times 1 u^{20} \mathrm{~m}^{-3} \cdot q_{0}=1.2 . q(a \cdot 2) \pm 1.3 . q^{\prime}(a \cdot 2)=11.4 . T_{1}=T_{e}=T$, with $T($ a 2$)=8$ kel. $T(0)=211$ kel. and wh the number of TF coils $n=12$. The gualut atwe resuls are unchanged using parameters for other machines in the range of TFTK t $s$ ITER. For the IXTOK pararieters, sne has $\tau_{s l} \simeq 1.15 \mathrm{sec}$. 5.. that $D=a^{2}\left(t \tau_{a}\right)=1.5 m^{2}$ sec or greater is urlacceptable

TABLE I: Alpha Transport Cuefficients

\begin{tabular}{|c|c|c|}
\hline Mechanism & Equation & Galue $\left(\mathrm{m}^{2} / \mathrm{sec}\right)$ \\
\hline symmetric neoclassical & $D_{b n-1} \simeq \nu_{s i}(q \rho)^{2} / t^{1 / 2}$ & 0.044 \\
\hline symmetric neoclassıcal & $\bar{D}_{b_{m-1}}=\nu_{s 1}(q \rho)^{2} / \epsilon^{3 / 2}$ & 0.46 \\
\hline superbarana (ripple) & $D_{s-t} \geq \nu_{-} r^{2} / a^{5} \delta^{1 / 2}$ & $1.8 \times 10^{-4}$ \\
\hline superbanana (ripple) & $D_{s b-r}=v_{-} r^{2} / \alpha^{2} \epsilon^{1 / 2}$ & $4.3 \times 10^{-3}$ \\
\hline banaja-drift (ripple) & $D_{b d} \simeq \nu_{1} \delta^{3 / 2} n^{9 / 4} q^{13 / 4} \rho R / \epsilon^{3 / 2}$ & $1.7 \times 10^{-2}$ \\
\hline stuchastic regime (ripple) & $D_{1} \simeq \epsilon^{1 / 2}\left(\pi / \Omega_{b}\right) v_{B}^{2}\left(J^{2} J^{2}\right)$ & \\
\hline.$M H U\{n=1=m .6=.01\}$ & & 39.6 \\
\hline$T F(n=12 . \delta=.1301)$ & & 4.75 \\
\hline electrostatic turbulence & $\bar{D}_{\varepsilon} \approx\left(\pi / \Omega_{b}\right) \dot{v}_{E}^{2}\left\langle J^{2} J^{2}\right\rangle$ & $0.51(0.022)=1.1 \times 11{ }^{-2}$ \\
\hline magnetic turbulence & $D_{b}=\left(\pi / \Omega_{b}\right)\left(v_{11} b\right)^{2}\left(J^{2} J^{2}\right)$ & $2.8(0.022)=6.2 \times 111^{-2}$ \\
\hline
\end{tabular}

Theorv exists for diffisive alpha transport due to axisymmetric ("necelassical" ${ }^{8.9}$ ripple, ${ }^{6.7 .10-12}$ and turbulent ${ }^{7,13-15}$ mechanisms. The first five of these are collisional mechanisms, all linear in either the slowing-down frequenc $v \nu_{s} \equiv \tau_{x}^{-i}$ or the perpendicular scattering frequency $\nu_{1}$. The last three (below the second double line) are collisionless. $D_{b n-1}$ and $D_{b n-1}$ are the 'fast' and 'slow' variants of the banana transport appropriate to alphas. the former applying when the alpha birth speed $v_{0}$ is so much greater than the critical speed $v_{b}$ that pitch-angle scattering may be neglected $\left.\epsilon\left(v_{0} / v_{b}\right)^{3}>1\right]$, and the latcer applving in the opposite limit $i \epsilon\left(v_{0} / v_{b}\right)^{3} \leq 1 !$ $D_{t-t}$ and $D_{t-r}$ are two limits of "superbanana" ripple transport, ${ }^{10,12}$ in which the geometric parameter $\alpha \equiv \epsilon /(n q \delta)$ is small enough that alphas can entrap in the ripple wells over a significant region of the poloidal plane. The quantity $\delta \equiv 6 B / B$ characterizes the magnitude of the field perturbations, due to WHD modes or to TF coils. Thus, in general. the field has the form $B=B_{0}[1 \div \delta \cos (m \theta-n \phi)]$. When $\delta$ is below the threshold value $\delta$, needed for the stochastic regime to be operative, the banana-centers of toroidally: trapped alphas can still drift due to the ripple, at the collisional "bananadrift" rate ${ }^{11} D_{b d}$. From Table $I_{1}$ one notes that, basically because alphas 
are nearlv collisicnless $\left(\nu_{s l}=6.5 \mathrm{sec}{ }^{i} . \nu_{-} \simeq 4.19 \mathrm{sec}^{-i}\right)$. these cullisional mechanusms are weak.

The o dlisiunal mechanisms having been elimunated, une is left with the "it , chasuc regime." ${ }^{-r}$ governed by $D_{s}$, and the magnetuc $\left(D_{b}\right)$ and electrostatic $\left(D_{E}\right)$ collisiondess turbulent mechanisms. Tu accusunt properly for the large grroradius and perpendicular drifts of alphas, and also (in the case if $D_{s}$ ). for the possibilitv of low- $\pi$. time-dependent ripple from MHD perturbatins, the appropriate $D$ for each of these has been generatized from Its form in earlier theorjes. ${ }^{6.13 .14}$ These "orbit-averaging" effects included bv the generalization are represented by the factor $\left\langle 0^{2} J^{2}\right\rangle \equiv\left\langle J_{l_{2}}^{2}\left(z_{g}\right) J_{l_{4}}^{2}\left(z_{b}\right)\right\rangle$ appearing in each of the three $D$ s the $J_{l}(z)$ are Bessol functions, phrsically crrresponding to the fraction of the gyro and bounce-period that a particle spends lorally resonant with the perturbations inducing the transport. Fur $D_{1}$. this fact or describes the "variable lingering period" during which a particle takes a radial step in the random- walk process.

The values given for $D_{E}$ and $D_{b}$ in Table 1 use current estirnates of the amplit udes of the turbulent fluctuations in the plasma interior $\bar{b} \equiv \bar{B}_{\mathrm{r}} / B \simeq$ $10)^{-i} \cdot \dot{0} \cdot T=111^{-2}$, and assume a turbulent spect rum characterized by perpendicular wave number $\lrcorner k_{-} \simeq 0.1 / \rho_{i}$, with $\rho_{i}$ the thermal ion gyroradius. One sees that the orbit-averaging factor (enclosed in angular brackets in Ta(3) 1) redures the turbulent mechanisms from the range where they would be of concern $\left(D \sim 1 \mathrm{~m}^{2} ; \mathrm{sec}\right)$ to a value not of concern $\left(D \sim 0.01 \mathrm{~m}^{2} / \mathrm{sec}\right)$.

In Table $I$, we consider two cases of $D_{2}$, one due to the ripple from an MIHD mode with $n=1=m$ and typical ripple amplitude $\delta \sim 0.01$, and the second due to TF ripple, so that $n=12 . m=0$, and typical ripple halfway out of $\delta \sim 0$.001. For both cases, $D$, is quite substantial. The low- $n$ case is especially large, and would represent essentially instantaneous loss of all trapped alphas, if the mechanism were operative. (However. as will be discussed shortly, the mechanism probably is not operacive, due to a verv large threshold for low- $n$ ripple.)

From the preceding, we conclude that one need only focus on transport from the stochastic mechanism. For the stochastic regime to be operative, a threshold condition on the ripple strength $\delta$ must hold, given for circular cross-section tokamaks by

$$
\delta>\delta_{1} \equiv(\epsilon / \pi n q)^{3 / 2} /\left(2 \rho q^{\prime}\right) .
$$

This mechanism is easily understood. Conservation of energy and magnetic moment implies a constant value of $B$ at the banana tip. The pres- 
ence of ripple causes a displacentent of the banana tip, both radially and in toroidal angle. When this displacement is comparalsle tu the ripple perind. the up motion becomes srichastic and the banana top moves vertically (conserving $B$ ) in a random ualk. until the irlot intersects the wall near the midplarte. The successive banana tip jusllus form an area-preserving map sunilar to the standard map ${ }^{6}$ and enset i, stochastic behavior can be estimated using the Chirikov overlap criterisn. giving Eq. (1) without the factor of 2 in the denominator. As in the case of the standard map. the actual stochastic threshold occurs at a value of $\varepsilon$ at least a factor of 2 smaller than the Chirikov estimate, and must be determined numerically. Equation (1) represents a reasonable approximation to the true threshold. The large threshold salue for small $n$ is simply due to the long ripple period, and consequently large tip displacement required to produce stochastic motion.

The nature of the loss process implies that particles make impart with the wall near the midplane. at a point on the orbit shortly before reaching maximum banana width. The mean distance below the midplane is pror portional to the mean tip displacement for a single bounce. Toroidally, the impart is more likely to orsilr where the field ripple causes an out ward drift. The guiding center $\operatorname{codes}^{i-3}$ give results consistent with this. Thus, while RIPLOS presently does not give detailed information about the alpha wall deposition distribution, but only the total lriss. it may also be possible to have it provide this more detaled surt of loss information.

For the case of TF rippte, even for $\delta \sim \delta_{\text {, }}$, the stochastic regime diffusion coefficient $D_{1}$ is large enough to be of concern. For the present sample parameters. for $r=a / 2$. one has $\delta_{s} \geq 2.2 \times 10^{-3}$ for $n=12$, so that (cf. Table I) $D_{s}(n=12)=23 \mathrm{~m}^{2} / \mathrm{sec}$. Due to the strong sensitivity bath of $D_{s}$ on $\delta$. and of $\delta$ on spatial position, $D$, grows rapidiv $\left(\tau_{a} \ll \tau_{l l}\right)$ as one moves radially outward from the boundary curve $C$ in the poloidal plane where $\delta=\delta$, (cf. Fig 2). Thus; a simple approximate rule for ajpha confinement may be given: banana-trapped alphas whose turning points are tn a regron where $\delta<\delta$, will be confined. while those with turring points in the regron $\delta>\delta$, will be lost in a time much less than $\tau_{d}$. (If the ripple is due to internal MHD activity, the second portion of this rule must be modified a bit: alphas with tursing points having $\delta>\delta$, will be rapidly moved to the boundary of this stochastic region.)

For low-n ripple, one notes from Table 1 that $D_{n}$, if operative, would expel alphas extremely rapidly from the regions of localization of the MHD modes inducing the transport. A combined scenario can be envisjoned, with 
MHD transporting alphas out from the central birth region, with TF-ripple taking wee to transport them to the plasma edge. However. since from Eq (1). $t, 2 n{ }^{3,2}$, one has $\{, i r-a: 2, n=11=11 .(185$, i.e. low $-n$ ripple has a verv large stochasuc threshold. (Low-n MIHD modes can typicaly produce ripple of a lew percent during a sawtuoth collapse, however, not far from this threshold.) Thus. as long as the MHD ripple is below this talue. the alpha ripple transport from this source should be small, governed by the collisional result $D_{b d}$. In the numerical results reported here we shall assume this, and consider only loss due to TF ripple.

RIPLOS reads numericallv generated equilibria, which can have arbitrary cross-sectional shape and pressure and current disíributions. Lsing a randomly generated birth distribution conforming to a chosen analytic form, it applies the simple loss cricerion given above, using the generalization of Eq. (1) to an arbitrary equilibrium. In Fig. 1 are shown the radial profiles used to clescribe (a)the alpha birth distribution, (b) safety factor $g(r)$, (c) $q^{\prime}(r)$. and $(d)$ the ripple strength $\delta$, and stochastic threshold $\delta_{0}$, for INTOR. $\delta$ was fit using the form

$$
\left.\delta=\delta_{0} \exp \left\{\left(R-R_{0}\right)^{2}-t z^{2}\right\}^{1 / 2} / w\right\}
$$

with $R_{0}=4.58 \mathrm{~m}, u^{\prime}=0.63 \mathrm{~m}$. and $b=0.25$, which can be made to match the ripple contours and ralues fairlv well in the domain with $\delta>5 \times 10^{-4}$ Values smaller than this are not relevant.

In Fig. 2 the loss and confinement regions are shown for INTOR parameters, assuming $\delta_{a} \equiv \delta(r=a, \theta=0) \simeq 0.0 \mathrm{i}$, and $q_{a} \equiv q(r=a) \simeq 2.5$. As $q_{a}$ or $\delta_{a}$ are increased. one sees from Eq. (1) that the position of $C$ moves radially inward and, particularly because of the strong $r$-dependence of the alpha birth profile, the loss fraction increases rapidly with $\delta_{a}$ and still more rapidly with qa, providing a simple explanation of the strong ga-dependence noted earlier by Tani, et al. ${ }^{2}$ The dependence on $q_{a}$ is of particular concern, because greater $q_{a}$ is more desirable from the standpoint of MHD stability, and also of remuining below the threshold $\beta_{\alpha}^{f b} \propto \boldsymbol{q}^{\prime}$ for direct (i.e., nondiffusive) loss due to fishbones. ${ }^{36}$

In Fig. 3 are shown benchmark comparisons of RIPLOS results (curves) with those from the ge code (individual points), for INTOR, and also for TFTR, plotting loss fraction versus $q_{a}$. The plots of Figs. 1 and 2 corre spond to the NTTOR point at $q_{\mathrm{a}}=2.5$. Each RIPLOS curve consists of ten points, each point coming from an ensemble of 40,000 particles. One notes that the RUPLOS and gC results are in agreement, to within better 
than a factor of 2 . The strong sensitivity of the results to the $q$ profule. a poorly known quantity. indicates that higher accuracy than this is nol worth the expense. The saluration of the loss fraction at large values of $g_{a}$ is due tu all trapped alphas being lost. As alreadv indicated. operatisn at higher $q$-values. preferable from an MHD-stability standpoint, is excluded by the requirement of acceptable alpha confunement. Moreuver, due to the very st rong $q_{a}$-dependence of $\varepsilon_{g}$. one can only gain slightly in the permissible $q_{c}$ by desigrung the tokamak with decreased $\delta_{\mathrm{n}}$, while keeping the loss fraction constant.

Summarizing, we have developed a new numerical tool (RIPLOS) for assessing total alpha loss in tokamaks of arbitrary cross section, which is many orders of magnitude faster than previous codes developed for studying this problem. and which yields results in reasonable agreement with those from the earlier codes. The code's simplicity is made possible by the realizatuon that stachastic ripple-transport should be the chief loss mechanism for alphas. and by making use of the threshold chararter of that mechanism. 


\section{References}

'R. B. White and M.S. Chance. Phys. Fluds 27. 245̄ (1984)

${ }^{2}$ k. Tanu. T.Takizuka. M.Azumi and H.kishumotu. Vucl.Fuston 5, 65 T (1983)

${ }^{3}$ L.M. Hively. Vucl, Fusion 24, if9 [1984).

${ }^{4}$ H.B.White.A.H.Bowzer, F.Goldston, H.Hay, J.Albert and C.F.F.Kamey. IAEA-CX-4!/T-3 (Vienna, 1983).

${ }^{5}$ K. Tani. T Takizuka and M.Azumi, US.Japan INTOR workshop, Jagova $(2 / 86)$

${ }^{6}$ R.J. Goldston. R.B.White and A.H.Booztr, Phys Rev. Lett. 4T, 647 (1481)

'H.E.Mrnick and K.E.Durall, P'rinceton Plasma Physics Laboratory Report tio PPPL.2E34 (1988)(to be published)

A Nocentini, M. Tessarotto and F.Enge!mann, Nucl. Fuszon 15, 359 (1975).

${ }^{9}$ P.J.Catto and A1. Tessarotto.SAIC-87/1826 (1987).

${ }^{10} \mathrm{P}$ N. Yushmanov. Vucl. Fuston 22, 315 (1982)

11 YaGoloborod'kó. Ya.I.Kolesnichenko and V.A.Yavorskij, Physicu Scripta T16, 46 (1987).

${ }^{12}$ H.E. Mynick and W.N.G. Hitrhon, Nucl.Fusion 24, 317 (1984).

${ }^{13}$ A. B. Rechester and M. N. Rosenbluth, Phys, Kev. Lett. 40. 38 (1978).

${ }^{14}$ K.T.Tsang, D.J.Sigmar and J.C.Whitson, Phys. Fluids 24, 151$) 8$ (1981).

${ }^{15}$ H.E.Mynick and J.A.Krommes, Phys. Fluids 23,1229 (198G).

${ }^{16}$ L.Chen, R.B. White and M.N.Rosenbluth, Phys.Rev.Lett. 52, 1122 (1984). 


\section{Figures}

FIC 1. IXTOR radial profiles for (a)aipha birth distribution. (b)safetv fac(ur $q \mid r)$. (c) $\varphi^{\prime}(r)$, and (d) ripple strength $\delta$. and stuchastic threstiold s.

FIG 2. I'ssition of the luss and confinement regions for lNTOR parameters, with $\varepsilon_{\mathrm{a}} \geq 0.01, q_{\mathrm{a}}=2.5$.

FIG. 3. Loss fraction versus $q_{a}$, comparing residts from the RIPLOS (curves) and guiding-center (individual points) codes. for INTOR and TFTR. 

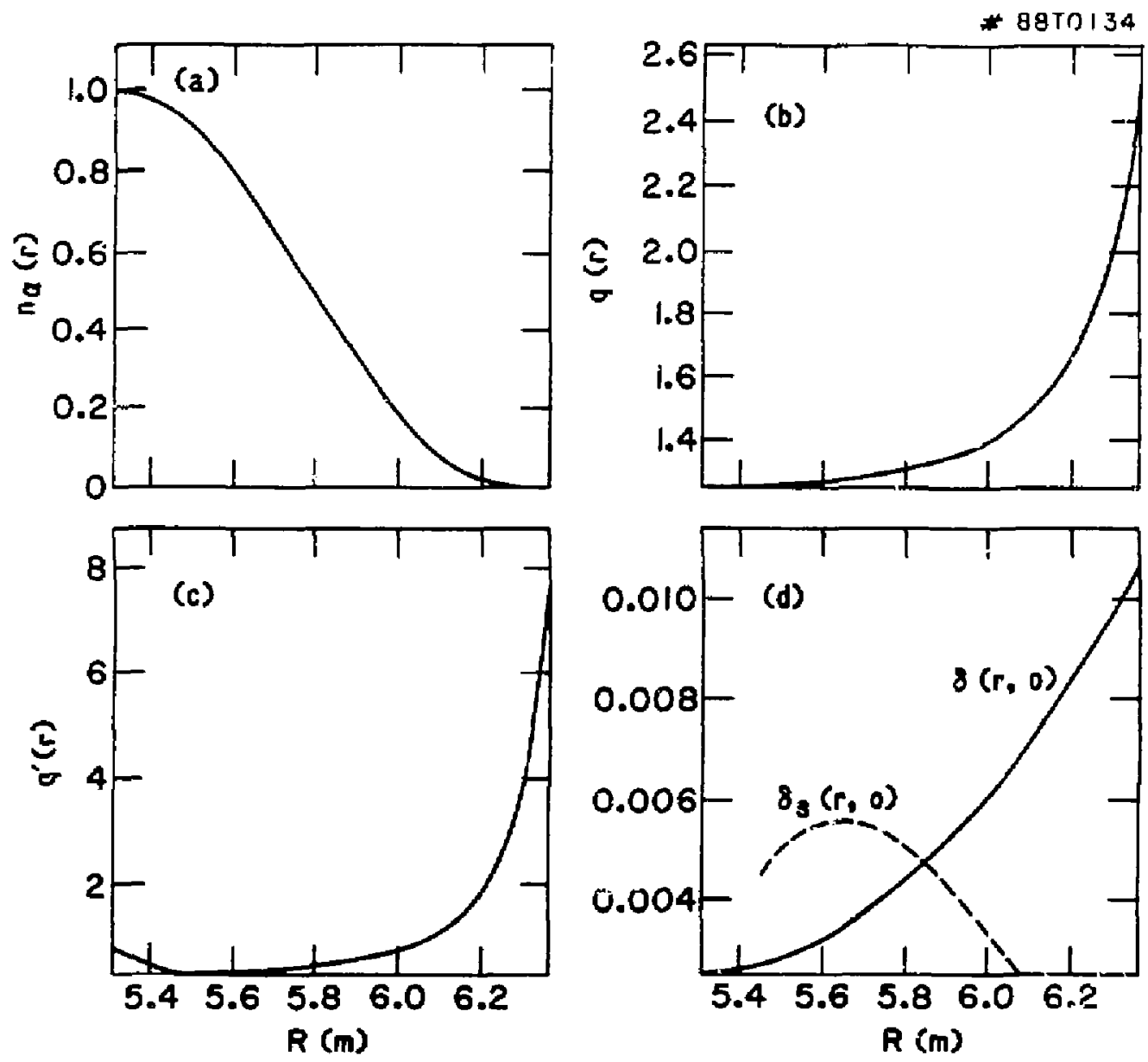

Fig. 1 


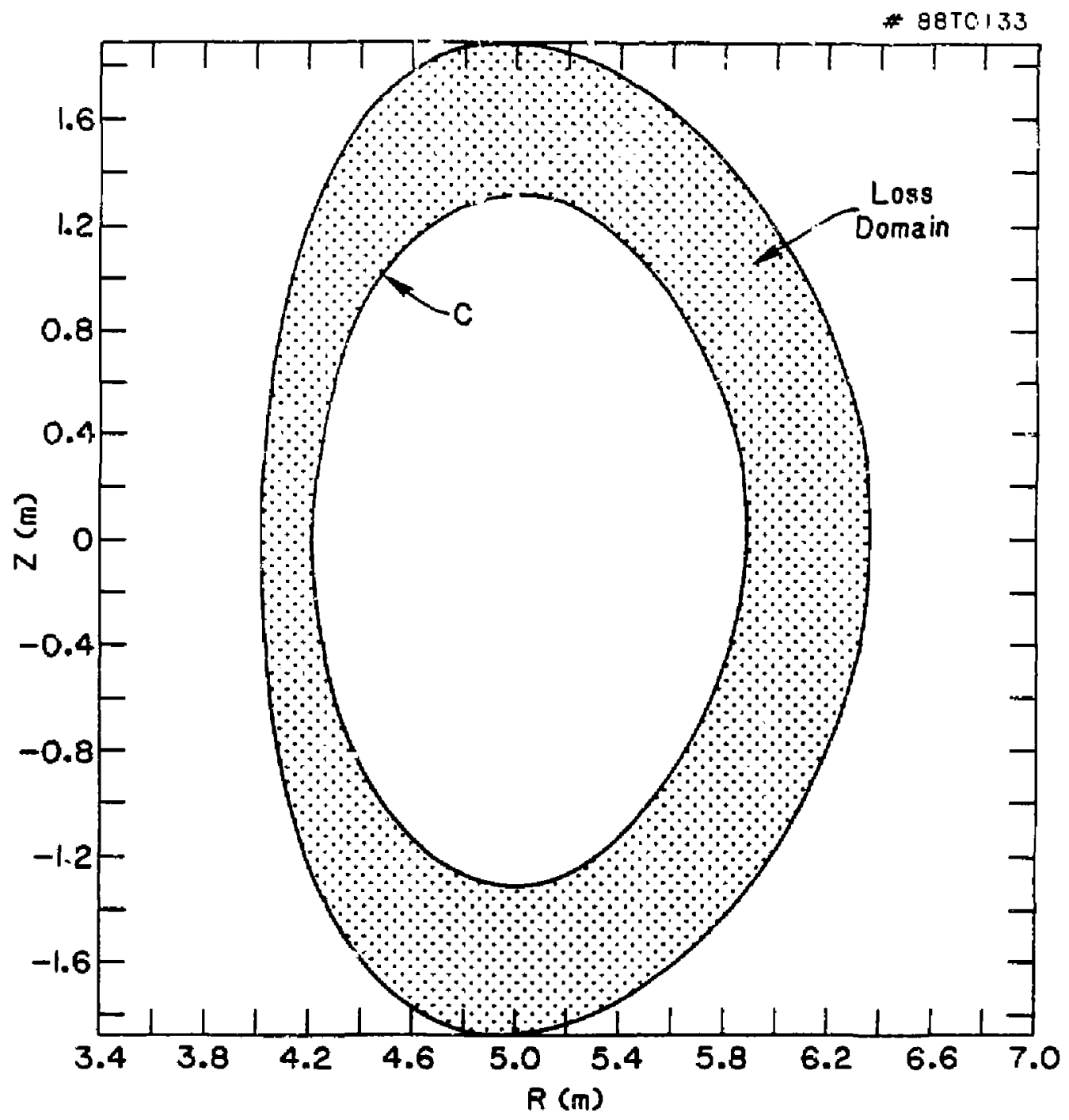

Eig. 2 


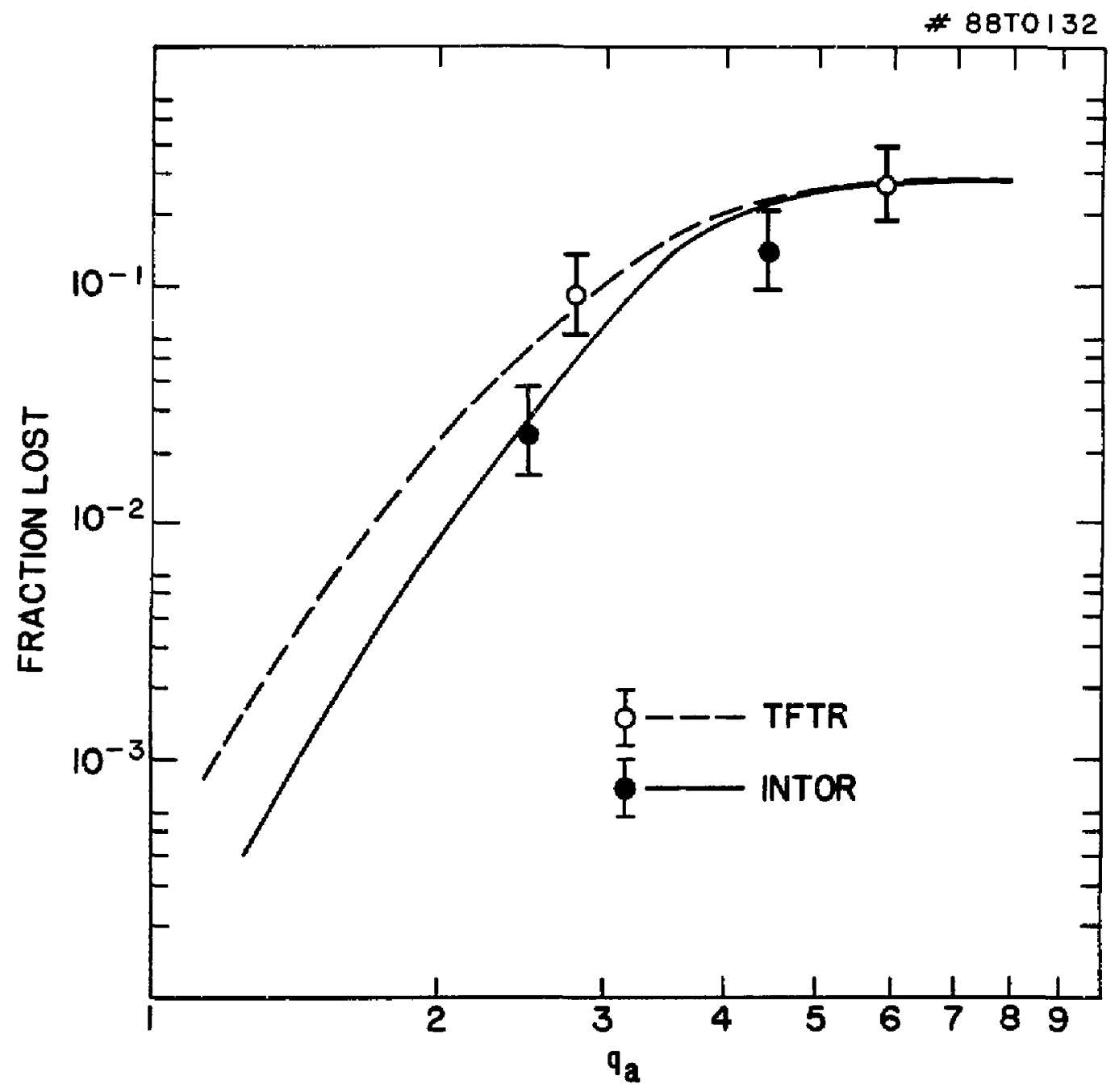

Fig. 3 